potentials factors related to tooth loss and loss of periodontal attachment.

Methods Cross-sectional study in 501 adults between 35 and 59 years, living in five different areas covered by the Community Health Agents Program/Family Health Strategy (CHAP/FHS), from July to October 2008. The oral health status information was obtained using indexes and criteria according Examiner's Manual SB Brazil project (2001). Descriptive and exploratory analyses were carried out and the differences in scores were tested by Pearson's $\chi^{2}$.

Results Near $50.0 \%$ of adults had tooth loss $(<20$ functional teeth present) and $17.7 \%$ had loss of periodontal attachment $\geq 6 \mathrm{~mm}$. Female had a greater tooth loss $(53.0 \%)$ and male had larger loss of periodontal attachment $\geq 6 \mathrm{~mm}$ (32.2\%). The adults with more than 45 years presented bigger tooth loss (67.2\%) as much as periodontal attachment $\geq 6 \mathrm{~mm}$ (24.3\%). Among the factors associated with tooth loss, can be pointed out no monthly individual income, low educational level and attending dentist when there is need $(p<0.001)$, while only low educational level was associated with loss of periodontal attachment $\geq 6 \mathrm{~mm}$ $(\mathrm{p}<0.001)$.

Conclusions The oral health status of adults was poor, which makes it one of the priorities of the primary care. Low socioeconomic status is commonly related to this condition, suggesting that improving the socioeconomic determinants reflect improvements in oral health.

\section{SP3-83 RELATIONSHIP BETWEEN CLINICAL DENTAL STATUS AND THE PERCEPTION OF ORAL HEALTH-RELATED QUALITY OF LIFE IN ADULTS, BRAZIL}

doi:10.1136/jech.2011.1429760.83

${ }^{1} \mathrm{~F}$ Floriano, ${ }^{*} \mathrm{C}$ Costa Mda, ${ }^{2} \mathrm{~A}$ Sheiham, ${ }^{2} \mathrm{G}$ Tsakos, ${ }^{3} \mathrm{M}$ I Vianna, ${ }^{3} \mathrm{M}$ B Cabral, ${ }^{1} \mathrm{G}$ Santos, ${ }^{3} \mathrm{M}$ dos Santos. ${ }^{1}$ ISC-UFBA, SSA, Bahia, Brazil; ${ }^{2}$ UCL, London, UK; ${ }^{3} \mathrm{FO}-$ UFBA, SSA, Bahia, Brazil

Introduction The objective of this study was to assess the impact of oral health-related quality of life (OHRQoL) in adults and to investigate the relationship between OHRQoL indicator and specific clinical dental measures.

Methods Cross-sectional survey was carried out of 501 adults between 35 and 59 years, living in five different areas covered by the Community Health Agents Program/Family Health Strategy (CHAP/FHS) in Salvador-Bahia, Brazil. Data were collected from July to October, 2008, through interviews and clinical examination that's used indexes and criteria according Examiner's Manual SB Brazil project (2001). OHRQoL was assessed through the General Oral Health Assessment Index (GOHAI). Data analysis used nonparametric tests (Mann-Whitney, Kruskal-Wallis and multiple linear regressions).

Results $51.1 \%$ of adults had tooth loss ( $<20$ functional teeth present), the GOHAI mean overall score was $29.4 \pm 4.7$ and $50.7 \%$ reported low levels of OHRQoL Female perceived a greater impact of oral health status in quality of life (GOHAI $=29.1 \pm 4.9)$. Concerns about oral health was the most frequently reported problem $(64.9 \%)$ while concerns about appearance $(53.7 \%)$ and oral impacts related to pain $(53.1 \%)$ were experienced by just over than half of participants. Tooth loss remained associated with worse perceptions of OHRQoL $(p<0.05)$ even after adjusted for age, gender, economic status, and loss of periodontal attachment.

Conclusions Poor oral health status increased the perceived impact of OHRQoL. Policies of reduction of oral health inequalities can be successful strategies to improve the quality of life of adults.
SP3-84 IMPACT OF ORAL HEALTH STATUS ON OUALITY OF LIFE IN ADULTS, BRAZIL

doi:10.1136/jech.2011.1429760.84

${ }^{1} \mathrm{~F}$ Floriano, ${ }^{*} \mathrm{C}$ Costa Mda, ${ }^{2} \mathrm{G}$ Tsakos, ${ }^{2} \mathrm{~A}$ Sheiham, ${ }^{3} \mathrm{M}$ I Vianna, ${ }^{3} \mathrm{M}$ B Cabral, ${ }^{3} \mathrm{M}$ dos

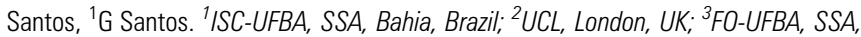
Bahia, Brazil

Introduction The objective of this study was to assess the prevalence of impacts of oral health status on quality of life among adults, Brazil. Methods A cross-sectional survey was carried out of 501 adults between 35 and 59 years, living in five different areas covered by the Community Health Agents Program/Family Health Strategy (CHAP/FHS) in Salvador-Bahia, Brazil. Data were collected from July to October, 2008, through interviews and clinical examination that's used indexes and criteria according Examiner's Manual SB Brazil project (2001). Data on the impact of oral health on the quality of daily living based upon the Oral Impacts on Daily Performance (OIDP) indicator. Exploratory analyses were tested by Pearson's $\chi^{2}$.

Results OIDP mean score was $12.1 \pm 6.3$ and $39.7 \%$ reported that their mouth affected their pattern of daily living. Oral impacts levels were lowest in subjects with the less loss of periodontal attachment. The most common oral impacts were on eating (20.6\%) followed by the ones related to aesthetics (smiling -16.4\%). Approximately 15\% of the adults had oral impacts on cleaning teeth and relaxing. Oral impacts were more prevalent among the female (19.3\%).

Conclusions This survey has shown that the oral status of adults fairly frequently affects their quality of life, and in particular, the ability to eat. Assess the oral health-related Quality of life promotes a more complex understanding of oral health, adds a subjective perspective to clinical interventions that's provide measures to improve the quality of life of patients.

\section{SP3-85 DETERMINANTS OF LIFETIME DENTAL PAIN IN TWO BRAZILIAN BIRTH COHORTS}

doi:10.1136/jech.2011.1429760.85

${ }^{1} \mathrm{M}$ Saraiva, ${ }^{1} \mathrm{H}$ Bettiol, ${ }^{2} \mathrm{~A}$ Silva, ${ }^{1} \mathrm{~T}$ Taglieri, ${ }^{1} \mathrm{~L}$ Taboga, ${ }^{1} \mathrm{M}$ A Barbieri. ${ }^{1}$ University of São Paulo, Ribeirão Preto, Brazil; ${ }^{2}$ Federal University of Maranhão, São Luiz, Brazil

Introduction Dental pain significantly impacts child's life. This study explores the determinants of lifetime dental pain (LDP) among children.

Methods Data from a follow-up of two birth cohorts in Brazil was used: Ribeirão Preto (RP) with one of highest Human Development Index (HDI) in the country and Sao Luiz (SL) one of the lowest. Follow-up data (1/3 of original cohort) was collected in 2004/5 when children from RP were 10/11 years old ( $n=790)$, and children from SL were $7 / 8$ years old $(n=673)$. Prevalence Ratios (PR) were calculated and multivariable analyzes was performed hierarchical modelling. Covariates were mother education, age, marital status, sex, skin colour, number of siblings and number of household members, mother's perception of child oral health, dental visit, and private dental insurance.

Results LDP prevalence in RP was $32.7 \%$, while in SL was $56.4 \%$. Common variables in the final model for the two cities were sex, mother's education, mother's perception of child oral health. For RP private health insurance (PR 0.67; 95\% CI 0.46 to 0.95 ) and marital status (PR 1.10; 95\% CI 0.87 to 1.40 ) were also included in the model. For SL visiting dentist in the past 2 years was positively associated with LDP (PR: 1.22 95\% CI 1.07 to 1.39 ) as well as the number of household members with children in families with more then five members showing PR 1.36 (95\% CI 1.03 to 1.79).

Conclusion The prevalence of LDP in both cohorts was relatively high and specially associated with family structure and socioeconomic position. 